\title{
Anatomía COMParada del tallo y de LA Raíz de las especies del género Neoevansia Marshall (Cactaceae)
}

\author{
Roberto Herrera-Cárdenas, Teresa Terrazas y Sofía Loza-Cornejo \\ Programa de Botánica, Colegio de Postgraduados, \\ Montecillo, Estado de México 56230, México. \\ Dirección electrónica winchi@colpos.colpos.mx
}

\begin{abstract}
Resumen. Se estudiaron los caracteres anatómicos de las especies de Neoevansia con el objetivo de evaluar la contribución de la anatomía en la sistemática del género. Se analizaron caracteres de los sistemas dérmico, fundamental y vascular de tallo y raíz y compararon con los de algunas especies de Peniocereus, Wilcoxia y Echinocereus. Los resultados mostraron que $N$. striata se caracteriza por presentar hipodermis no colenquimatosa de un solo estrato y punteaduras intervasculares alternas en elementos de vaso del xilema secundario; mientras que $N$. lazaro-cardenasii y $N$. zopilotensis se distinguen anatómicamente entre sí por el tamaño de las células epidérmicas y el grosor de la hipodermis. El incremento en volumen en las raíces tuberosas se relaciona principalmente a las divisiones del parénquima radial. Neoevansia lazaro-cardenasii, N. zopilotensis y $N$. striata comparten con Peniocereus la presencia de madera fibrosa y fibras sobre el floema. Los resultados apoyan las propuestas de varios autores de transferir a las especies de Neoevansia al género Peniocereus.

Palabras clave: Neoevansia, Cactaceae, Cactoideae, anatomía del tallo, raíz tuberosa.

Abstract. Anatomical characters of Neoevansia species were studied to gain insight in their contribution to the systematics of the genus. Characters of the dermal, fundamental, and vascular systems of the stem and root were evaluated and compared with several species of Peniocereus, Wilcoxia, and Echinocereus. The results revealed that $N$. striata is characterized by a noncollenchymatous unistratified hypodermis and alternate intervascular pits in vessel elements in the secondary xylem; while $N$. lazaro-cardenasii and $N$. zopilotensis can be distinguished by their epidermic cell size and hypodermis width. The increment in volume in Neoevansia tuberous roots is mainly related to cell divisions in rays. Neoevansia lazaro-cardenasii, N. zopilotensis, and N. striata share with Peniocereus the fibrous wood and the phloem fiber cap. The results support the proposals of several authors to transfer the species of Neoevansia to the genus Peniocereus.
\end{abstract}

Key words: Neoevansia, Cactaceae, Cactoideae, stem anatomy, tuberous root.

$\mathbf{L}$ os caracteres anatómicos son empleados frecuentemente con propósitos sistemáticos porque constituyen evidencias adicionales a las morfológicas, que pueden ser esenciales en la solución de problemas taxonómicos (Bailey, 1954). De manera particular, la anatomía del xilema secundario y la anatomía de los tallos jóvenes, contribuyen a ubicar taxa de afinidades inciertas (Dickison, 1989). En la actualidad son escasos los estudios con este enfoque en la familia Cactaceae (Boke, 1959; Loza-Cornejo y Terrazas, 1996; Nyffeler y Eggli, 1997; Mauseth et al. 1998), a pesar del alto número de géneros endémicos en el país, como es Neoevansia.
El género Neoevansia se caracteriza por tener raíces con numerosos tubérculos suaves (figuras 1,3); tronco leñoso, ramas delgadas erectas a trepadoras (figura 2); frecuentemente haptotrópicas, con superficie glabra y rugosa (Marshall, 1941). Costillas en número de 6 a 20, de sección cuneiforme, de cantos planos, convexos, separadas por un surco muy angosto. Aréolas pequeñas, circulares a elípticas. Espinas pequeñas. Flores grandes, tubular infundibuliformes, nocturnas y fragantes. Fruto obpiriforme a casi ovoide (figura 4). Semilla negra, brillante, tuberculada o finamente foveolada (Bravo-Hollis, 1978). El género comprende tres especies: N. striata (Brandegee) Sánchez-Mej. de 


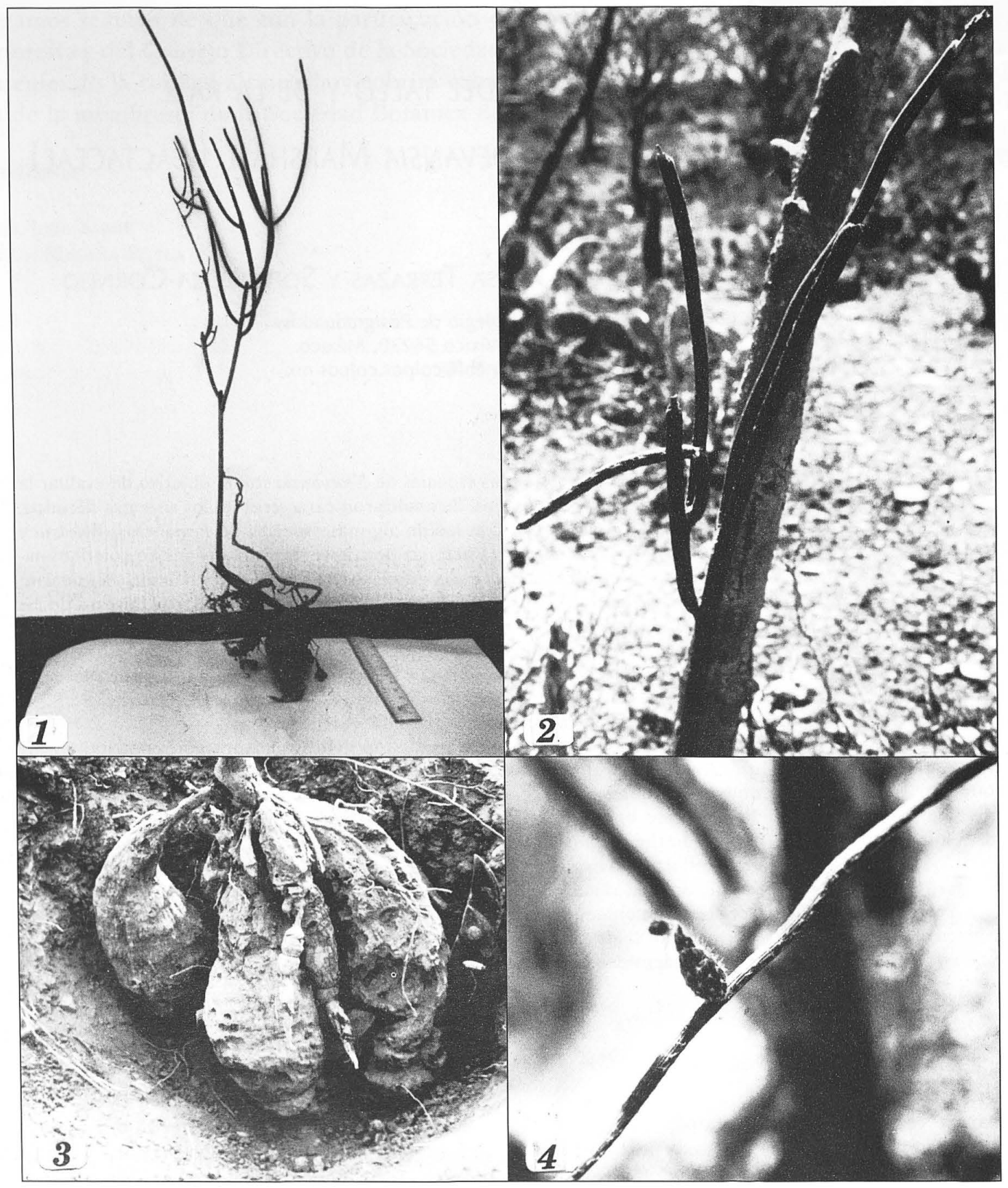

Figuras 1-4. Morfología de las especies de Neoevansia. 1. N. lazaro-cardenasii. Planta completa. 2-4. N. zopilotensis. 2. Ramas. 3. Raíces tuberosas. 4. Fruto.

Arizona, Baja California, Sonora y Sinaloa, N. zopilotensis J.Meyrán conocida del Cañón del Zopilote, Guerrero (Sánchez-Mejorada, 1973) y N. lazaro-cardenasii J.L.Contr. et al. endémica de la cuenca baja del río Balsas de Guerrero y Michoacán (Contreras et al.
1980). Varios autores consideran a Neoevansia como sinónimo de Peniocereus (Hunt y Taylor, 1990; Barthlott y Hunt, 1993). Sin embargo, ninguno de estos autores ubican a estas especies en alguno de los subgéneros de Peniocereus propuestos por Sánchez-Mejorada 
(1974). Ante esta perspectiva se estudió el género Neoevansia con la finalidad de evaluar la contribución de la anatomía en la sistemática de este taxon con los objetivos de conocer si existen caracteres anatómicos en las especies de Neoevansia que permitan distinguirlas y si Neoevansia puede considerarse un género con características anatómicas exclusivas al compararlo con especies de Peniocereus, Wilcoxia y Echinocereus.

\section{Materiales y métodos}

La recolección de las especies de Neoevansia se realizó en los estados de Sonora, Sinaloa, Michoacán y Guerrero (cuadro 1). En cada localidad se recolectaron dos o tres individuos completos por especie. Una vez extraído el individuo (figura 1), el tallo se fraccionó en región basal, media y apical, así como en tres o cuatro niveles de la raíz tuberosa y no tuberosa con el propósito de observar la variación estructural a lo largo del tallo y de la raíz. Las porciones de tallo y raíz se fijaron en FAA (Johansen, 1940). Parte del material recolectado se depositó en el HerbarioHortorio del Programa de Botánica del Colegio de Postgraduados (CHAPA). Los tejidos primarios (epidermis, córtex, haces vasculares, médula) y secundarios (xilema, floema, peridermis) se procesaron siguiendo la microtecnia presentada en Loza-Cornejo y Terrazas (1996). Las 25 mediciones por carácter por individuo se realizaron con un analizador de imágenes IMAGE-Pro Plus versión 3.1 (Media Cybernetics, 1997), adaptado a un microscopio Olympus BX-50. A las 15 variables cuantitativas se les aplicó un análisis de varianza (SAS Institute, 1989) seguido de un análisis de comparación de medias de Tukey con objeto de evaluar la existencia de diferencias estadísticamente significativas entre especies para los caracteres cuantificados. Las características utilizadas en la comparación de las especies de Echinocereus, Peniocereus y Wilcoxia con las de Neoevansia se tomaron de la literatura.

\section{Resultados}

A continuación se presenta la descripción de los caracteres anatómicos del tejido primario y secundario del tallo, así como las características de la raíz tuberosa y no tuberosa a nivel genérico. En los cuadros 2 y 3 se sintetizan los valores de los caracteres cuantitativos para tallo y madera, respectivamente y en el cuadro 4 se comparan los caracteres más distintivos del sistema dérmico y xilema secundario de las especies de Neoevansia con los de algunas especies de los géneros Echinocereus, Peniocereus y Wilcoxia. No se considera en esta comparación los caracteres anatómicos cuantitativos porque a la fecha no existe información disponible para todas las especies.

\section{Descripción anatómica del tallo}

Tejido primario. Cutícula y epidermis. La cutícula es lisa; tiene un espesor que varía de $1.18 \pm 0.31 \mu \mathrm{m}$ en $N$. lazaro-cardenasii hasta $2 \pm 0.30 \mu \mathrm{m}$ en $N$. striata (cuadro 2). Las células epidérmicas presentan forma rectangular en corte transversal y tienen una papila unicelular (figuras 5,6); en $N$. striata miden $12 \pm 2 \mu \mathrm{m}$ de ancho por $17 \pm 3 \mu \mathrm{m}$ de largo, mientras que en $N$. lazarocardenasii son de $52 \pm 17 \mu \mathrm{m}$ de ancho por $145 \pm 61 \mu \mathrm{m}$ de largo, presentándose diferencias estadísticamente significativas entre especies (cuadro 2). En vista superficial las células epidérmicas tienen paredes anticlinales rectas. Los estomas están restringidos a las depresiones de las costillas, son del tipo paracítico y se ubican al mismo nivel de las células epidérmicas adyacentes (figuras 6,7).

Hipodermis. Es biestratificada y colequimatosa en $N$. lazaro-cardenasii y N. zopilotensis (figuras 6, 8), mientras que en $N$. striata consiste solamente de un estrato de células con pared delgada. El grosor de la hipodermis fluctúa desde $16 \pm 5 \mu \mathrm{m}$ en $N$. striata hasta $50 \pm 16 \mu \mathrm{m}$ en $N$. zopilotensis (cuadro 2).

Cuadro 1. Localidades de recolecta de las especies de Neoevansia

\begin{tabular}{lll}
\hline Especie & Localidad & Individuos recolectados (colector) \\
\hline N. lazaro-cardenasii & $\begin{array}{l}\text { Infiernillo, Mich. } \\
\text { Palo Pintado, Mich. }\end{array}$ & 2 (Terrazas 395, 396) \\
& Culiacán, Sin. & 1 (Terrazas 461) \\
N. striata & Guasimas, Son. & 2 (Terrazas 413, 414) \\
& Río Mezcala, Gro. & 3 (Terrazas 305-307) \\
N. zopilotensis & Venta Vieja, Gro. & 3 (Terrazas 310-312) \\
\hline
\end{tabular}




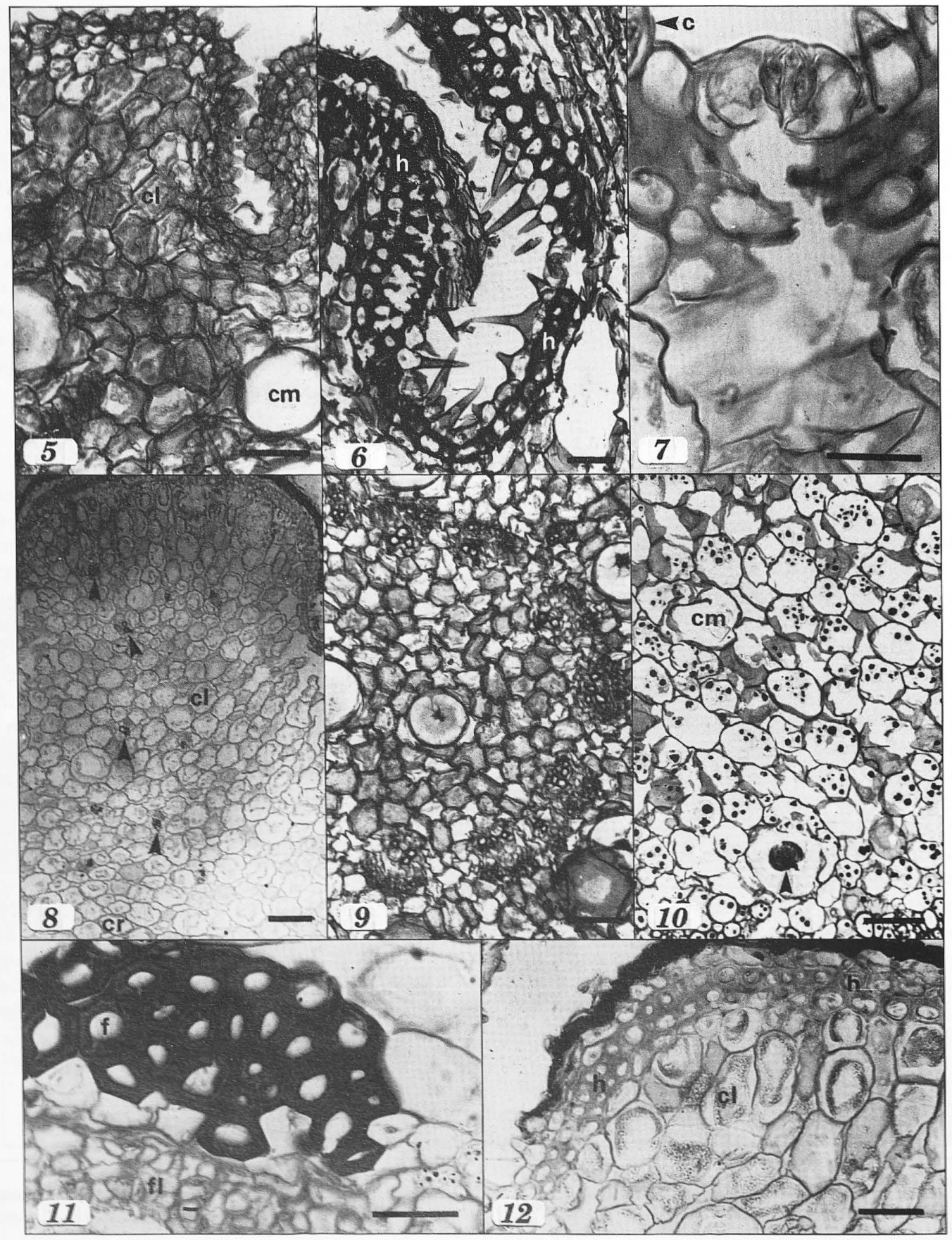

Figuras 5-12. Caracteres anatómicos del tallo. Cortes transversales. 5. N. zopilotensis. Epidermis y córtex. 6. N. lazarocardenasiz. Papilas, estoma e hipodermis. 7. N. zopilotensis. Estoma. 8. N. zopilotensis. Córtex clorenquimatoso y reservante; haces corticales (flechas). 9. N. zopilotensis. Estela. 10. N. zopilotensis. Médula con abundantes granos de almidón, células de mucilago y cristal (flecha). 11. N. zopilotensis. Fibras sobre el floema. 12. N. zopilotensis. Peridermis con felema colapsado. $\mathrm{c}=$ cutícula, $\mathrm{cl}=$ clorénquima, $\mathrm{cm}=$ célula de mucílago, $\mathrm{cr}=$ córtex reservante, $\mathrm{f}=$ fibra, $\mathrm{fl}=$ floema, $\mathrm{h}=$ hipodermis. Escala: 5, 6, 9, $12=100 \mu \mathrm{m}, 7,11=50 \mu \mathrm{m}, 8,10=200 \mu \mathrm{m}$. 
Córtex. Se caracteriza por tener dos zonas, clorénquima y reservante (figura 8) y mide de 0.61 a $1.67 \mathrm{~mm}$ en espesor (cuadro 2). El clorénquima es la región más próxima a la hipodermis, con células de forma rectangular, en disposición radial que al alejarse de la hipodermis pierden su arreglo; contienen gran cantidad de cloroplastos y presentan algunos cristales esféricos. Las células del parénquima reservante son de forma isodiamétrica con paredes onduladas y escasos granos de almidón. En ambas zonas del córtex se presentan células de mucílago y haces vasculares colaterales (figura 8), denominados haces corticales por Mauseth y Sajeva (1992). En el clorénquima las células de mucílago son abundantes, grandes y ocasionalmente fusionadas; mientras que escasas y pequeñas en la región reservante. El xilema de los haces corticales presenta de uno a dos vasos en $N$. lazaro-cardenasii y $N$. striata y hasta cinco en $N$. zopilotensis. El floema está constituido por elementos de tubo criboso y células acompañantes, ambos con forma angulosa y algunas células de parénquima.

Xilema y floema primario. La estela consiste de 15 a 35 haces colaterales (figura 9). En cada haz vascular, el xilema presenta además de células de parénquima de uno a tres vasos en $N$. lazaro-cardenasii y $N$. striata hasta seis en $N$. zopilotensis. Los elementos de vaso del xilema primario tienen pared secundaria con engrosamientos helicoidales (figura 17) y placa de perforación simple. El floema se compone por elementos de tubo criboso, células acompañantes y parénquima floemático. Los elementos de tubo criboso tienen forma angulosa en corte transversal y placa cribosa simple; las células acompañantes son más o menos triangulares.

Médula. Varía en diámetro de 0.19 a $1.83 \mathrm{~mm}$ en la parte media del tallo de las tres especies; solamente se detectaron diferencias estadísticamente significativas entre $N$. striata y las otras dos especies (cuadro 2). En general, las células tienen forma isodiamétri- ca y contienen abundantes granos de almidón. Los haces vasculares están ausentes en esta región y las células de mucílago son escasas, con excepción de $N$. zopilotensis (figura 10).

Tejido secundario. Peridermis. En la parte basal del tallo la epidermis es reemplazada por una peridermis unidireccional (figuras 6,12,15). El felógeno se origina de las células epidérmicas en las elevaciones de las costillas y se extiende hacia las depresiones. El felema se caracteriza por presentar las células adyacentes al felógeno de pared delgada, suberificada, de forma rectangular, a veces colapsadas radialmente. Dichas células conforman de uno a seis estratos que se alternan con otros de dos a cuatro células de pared gruesa, lignificada, también de forma rectangular y algunas ocluidas por taninos (figura 15). No se presenta felodermis.

Floema secundario. Se distingue floema no colapsado y colapsado. En el floema no colapsado los tubos cribosos y células acompañantes forman grupos separados por células de parénquima floemático. El floema colapsado se observa en capas separadas por células de parénquima floemático de forma irregular. Sobre el floema colapsado se establecen paquetes de fibras septadas y nucleadas, formando grupos de cinco a más de 20 células (figura 11).

Xilema secundario. La porosidad es difusa (figura 13), no se distinguen anillos de crecimiento en $N$. striata; sin embargo en $N$. lazaro-cardenasii y $N$. zopilotensis dichos anillos son distintivos por diferencias en el grosor de la pared de las fibras y mayor abundancia de parénquima en la madera temprana. Los vasos varían de 7 a $11 / \mathrm{mm}^{2}$ y se arreglan más del $70 \%$ solitarios y el resto en hileras radiales o grupos de dos a cuatro vasos. El diámetro tangencial de los vasos varía de $20 \pm 4 \mu \mathrm{m}$ en $N$. lazaro-cardenasii hasta $30 \pm 6 \mu \mathrm{m}$ en $N$. zopilotensis (cuadro 3). La longitud de los elementos

Cuadro 2. Valores de la media y una desviación estándar para los caracteres cuantitativos del tallo de las especies de Neoevansia estudiadas. Letras diferentes indican diferencias estadísticamente significativas (Tukey, $P<0.05)$

\begin{tabular}{lccc}
\hline Carácter anatómico & N. lazaro-cardenasii & N. striata & N. zopilotensis \\
\hline Grosor de la cutícula $(\mu \mathrm{m})$ & $1.18 \pm 0.27^{\mathrm{a}}$ & $2.0 \pm 0.30^{\mathrm{a}}$ & $1.18 \pm 0.31^{\mathrm{a}}$ \\
Largo de la célula epidérmica $(\mu \mathrm{m})$ & $145 \pm 61^{\mathrm{a}}$ & $17 \pm 3^{\mathrm{b}}$ & $47 \pm 17^{\mathrm{c}}$ \\
Ancho de la célula epidérmica $(\mu \mathrm{m})$ & $52 \pm 17^{\mathrm{a}}$ & $12 \pm 2^{\mathrm{b}}$ & $24 \pm 8^{\mathrm{c}}$ \\
Grosor de la hipodermis $(\mu \mathrm{m})$ & $32 \pm 5^{\mathrm{a}}$ & $16 \pm 5^{\mathrm{b}}$ & $50 \pm 16^{\mathrm{c}}$ \\
Grosor del córtex en cresta de la costilla $(\mathrm{mm})$ & $1.05 \pm 0.16^{\mathrm{a}}$ & - & $1.14 \pm 0.53^{\mathrm{a}}$ \\
Diámetro de la médula $(\mathrm{mm})$ & $1.11 \pm 0.10^{\mathrm{a}}$ & $0.24 \pm 0.05^{\mathrm{b}}$ & $1.44 \pm 0.39^{\mathrm{a}}$ \\
\hline
\end{tabular}


de vaso fluctúa de $193 \pm 28 \mu \mathrm{m}$ en $N$. striata a $243 \pm 27 \mu \mathrm{m}$ en $N$. zopilotensis. La placa de perforación es simple con orientación transversal; las punteaduras intervasculares y las punteaduras radio-vaso son escalariformes y pseudoescalariformes en $N$. lazaro-cardenasii y $N$. zopilotensis, pero alternas en $N$. striata. Las fibras son libriformes nucleadas, septadas o no septadas, con punteaduras simples en las paredes radiales (figura 17). La longitud fluctúa de 188 a $801 \mu \mathrm{m}$, con una media de $271 \pm 18 \mu \mathrm{m}$ en $N$. striata hasta $466 \pm 105 \mu \mathrm{m}$ en $N$. zopilotensis (cuadro 3). El diámetro fluctúa de
9 a $19 \mu \mathrm{m}$ y el grosor de la pared de dos a cinco $\mu \mathrm{m}$; no se detectaron diferencias estadísticamente significativas para este carácter (cuadro 3). El parénquima axial es paratraqueal escaso (figura 16), con dos células por serie parenquimática, con contenidos escasos. Los radios son heterogéneos (figura 14); no hay radios uniseriados y los biseriados son raros. Se reconocen radios multiseriados primarios y secundarios. Los primarios se extienden desde la médula hasta el cámbium vascular y los secundarios se inician a diferentes distancias de la médula (figura 13). La altura

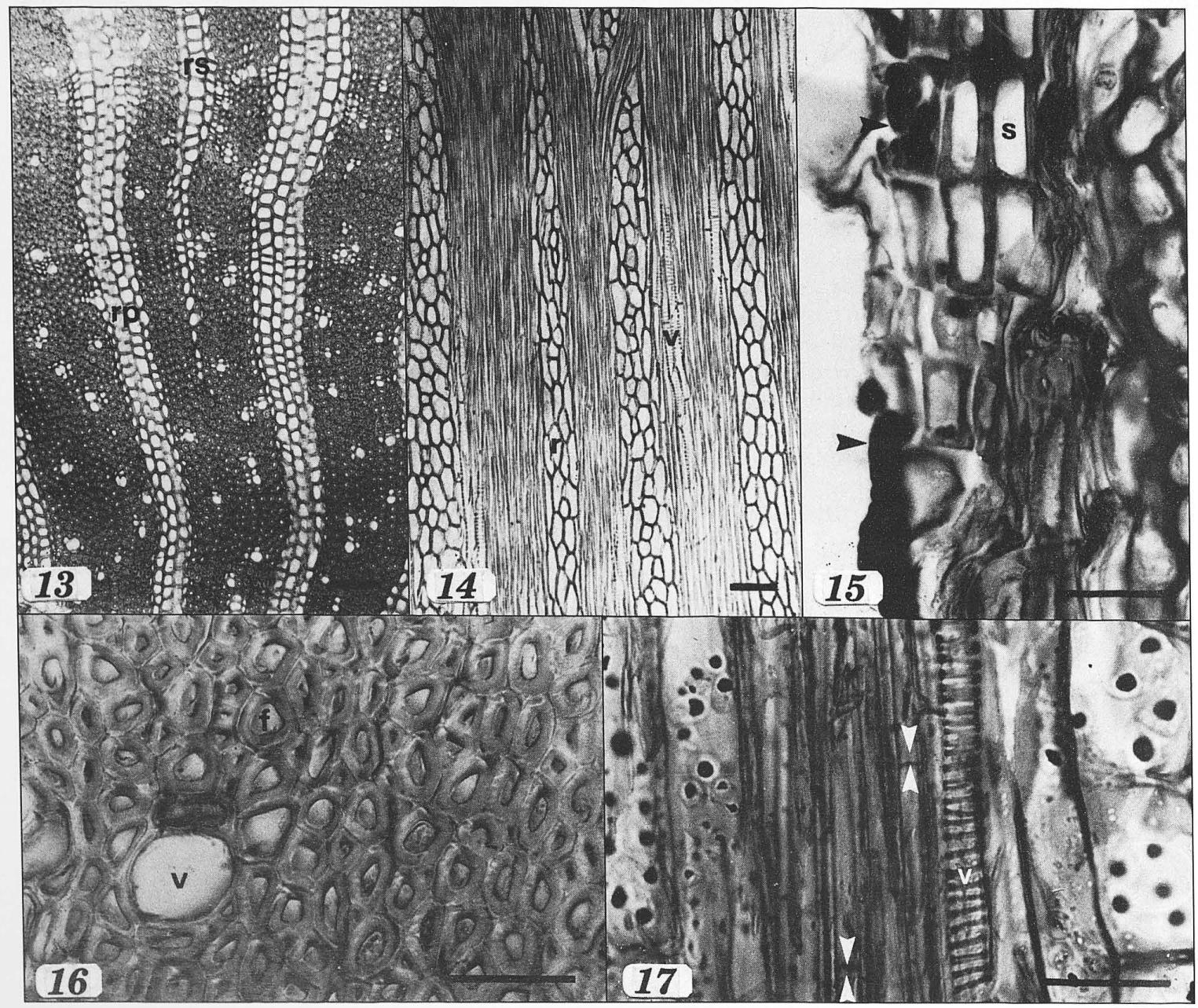

Figuras 13-17. Xilema secundario y peridermis. 13, 15, 16. Cortes transversales. 13. N. zopilotensis. Porosidad difusa, radios primarios y secundarios. 14. N. lazaro-cardenasii. Corte tangencial. Radios multiseriados. 15. N. lazaro-cardenasii. Peridermis, células del felema colapsadas y no colapsadas de pared gruesa. 16. N. lazaro-cardenasii. Fibras y vaso. 17. N. lazaro-cardenasii. Corte radial. Elementos de vasos, fibras septadas (flechas) y células de parénquima con granos de almidón. $\mathrm{f}=$ fibras; $\mathrm{r}=$ radios; $\mathrm{rp}=$ radios primarios; $\mathrm{r}$ = radios secundarios; $\mathrm{s}=\mathrm{súber} ; \mathrm{v}=\mathrm{vaso}$. Escala: $13,14=200 \mu \mathrm{m}, 15-17=50 \mu \mathrm{m}$. 
de los radios primarios varía de $1.82 \pm 0.41 \mathrm{~mm}$ en $N$. lazaro-cardenasii hasta $2.17 \pm 0.51 \mathrm{~mm}$ en $N$. zopilotensis y en anchura de $55 \pm 13 \mu \mathrm{m}$ en $N$. striata hasta $110 \pm 40$ $\mu \mathrm{m}$ en $N$. zopilotensis (cuadro 3 ) con tres a cinco series en la parte más ancha (figura 14). Los radios secundarios en las tres especies son más cortos y angostos que los primarios (cuadro 3). En ambos tipos de radios las células del cuerpo y de los márgenes son cuadradas y erectas, regularmente la porción marginal está formada por una o dos células. La abundancia de granos de almidón varía entre individuos.

\section{Descripción anatómica de la raíz}

Únicamente se estudiaron raíces con crecimiento secundario. La raíz tuberosa y no tuberosa comparten características anatómicas, por lo que se resaltan a continuación sus diferencias anatómicas.

Peridermis. Tiene origen y estructura semejante a la del tallo. Se observan de uno a dos estratos de células de felógeno y el número de estratos del felema tiende a ser menor que en el tallo (figuras 18,22,23).

Córtex. Entre la peridermis y el floema de la raíz tuberosa se presentan células de parénquima de forma isodiamétrica que se colapsan radialmente cerca de la peridermis (figura 18); mientras que en la raíz no tuberosa las células de parénquima son de forma rectangular y cuadrada (figura 22). Células de mucílago y haces corticales solamente se observaron en la raíz tuberosa. Los haces vasculares corticales son colaterales y pequeños. Escasos cristales se encuentran en el lumen de algunas células de mucílago.

Tejido vascular. El cámbium vascular forma un anillo completo (figura 19) en la raíz tuberosa y se distin- gue por presentar de uno a tres estratos de células de forma rectangular arregladas en hileras radiales. El xilema secundario se caracteriza porque los elementos de vaso están distribuidos en hileras radiales uni o bicelulares (figuras 19,21), que se alternan con células de parénquima y escasas fibras. Las fibras cuando presentes, se ubican cerca de la región central de la raíz. El parénquima radial separa las hileras de vasos y sus células tienen paredes angulosas en corte transversal (figura 19). Los radios son altos (>25 mm), formados de células erectas con abundantes granos de almidón y células de mucílago que contienen pequeños cristales. El floema secundario también se dispone en hileras radiales uni o biseriadas (figuras 19,21 ), éste se presenta no colapsado y colapsado. El primero, se ubica adyacente al cámbium vascular y se caracteriza por los tubos cribosos de forma rectangular, las células acompañantes y el parénquima floemático (figura 20); en el segundo se colapsan la mayoría de las células, excepto las de parénquima que se expanden (figura 19). Tanto los radios xilemáticos como floemáticos presentan divisiones periclinales y anticlinales que contribuyen distintivamente al aumento en diámetro de la raíz tuberosa. El xilema secundario en la raíz no tuberosa es semejante al del tallo, pero los vasos tienden a ser más numerosos y de mayor diámetro, las fibras son libriformes y el parénquima paratraqueal escaso (figuras 22,23). El floema secundario también es semejante al del tallo, sin embargo, el floema colapsado ocupa una mayor proporción del tejido de la raíz (figura 23) y los radios tanto xilemáticos como floemáticos se expanden ligeramente junto con la región central de la raíz.

Región central de la raiz. Con el incremento del diámetro de la raíz tuberosa se observa proliferación del parénquima del metaxilema. Esta región central se

Cuadro 3. Valores de la media y una desviación estándar para los caracteres cuantitativos del xilema secundario de las especies de Neoevansia estudiadas. Letras diferentes indican diferencias estadísticamente significativas (Tukey, $\mathrm{P}<0.05$ )

\begin{tabular}{|c|c|c|c|}
\hline Carácter anatómico & N. lazaro-cardenasii & N. striata & N. zopilotensis \\
\hline Diámetro del vaso $(\mu \mathrm{m})$ & $20 \pm 4^{a}$ & $24 \pm 3^{\text {ab }}$ & $30 \pm 6^{b}$ \\
\hline Longitud del elemento de vaso $(\mu \mathrm{m})$ & $220 \pm 93^{a}$ & $193 \pm 28^{b}$ & $243 \pm 27^{a}$ \\
\hline Longitud de fibra $(\mu \mathrm{m})$ & $471 \pm 83^{a}$ & $271 \pm 18^{b}$ & $466 \pm 105^{a}$ \\
\hline Diámetro de fibra $(\mu \mathrm{m})$ & $11 \pm 1^{\mathrm{a}}$ & $16 \pm 2^{b}$ & $15 \pm 2^{b}$ \\
\hline Grosor de la pared de fibra $(\mu \mathrm{m})$ & $3 \pm 0.5^{a}$ & $3 \pm 0.6^{a}$ & $4 \pm 1^{\mathrm{a}}$ \\
\hline Altura radio primario $(\mathrm{mm})$ & $1.82 \pm 0.41^{\mathrm{a}}$ & $2.05 \pm 0.11^{\mathrm{a}}$ & $2.17 \pm 0.51^{\mathrm{a}}$ \\
\hline Anchura radio primario $(\mu \mathrm{m})$ & $98 \pm 19^{a}$ & $55 \pm 13^{b}$ & $110 \pm 40^{\mathrm{a}}$ \\
\hline Altura radio secundario (mm) & $0.99 \pm 0.41^{\mathrm{a}}$ & $1.18 \pm 0.09^{a}$ & $0.95 \pm 0.36^{\mathrm{a}}$ \\
\hline Anchura radio secundario $(\mu \mathrm{m})$ & $110 \pm 40^{\mathrm{a}}$ & $75 \pm 17^{a}$ & $110 \pm 39^{a}$ \\
\hline
\end{tabular}




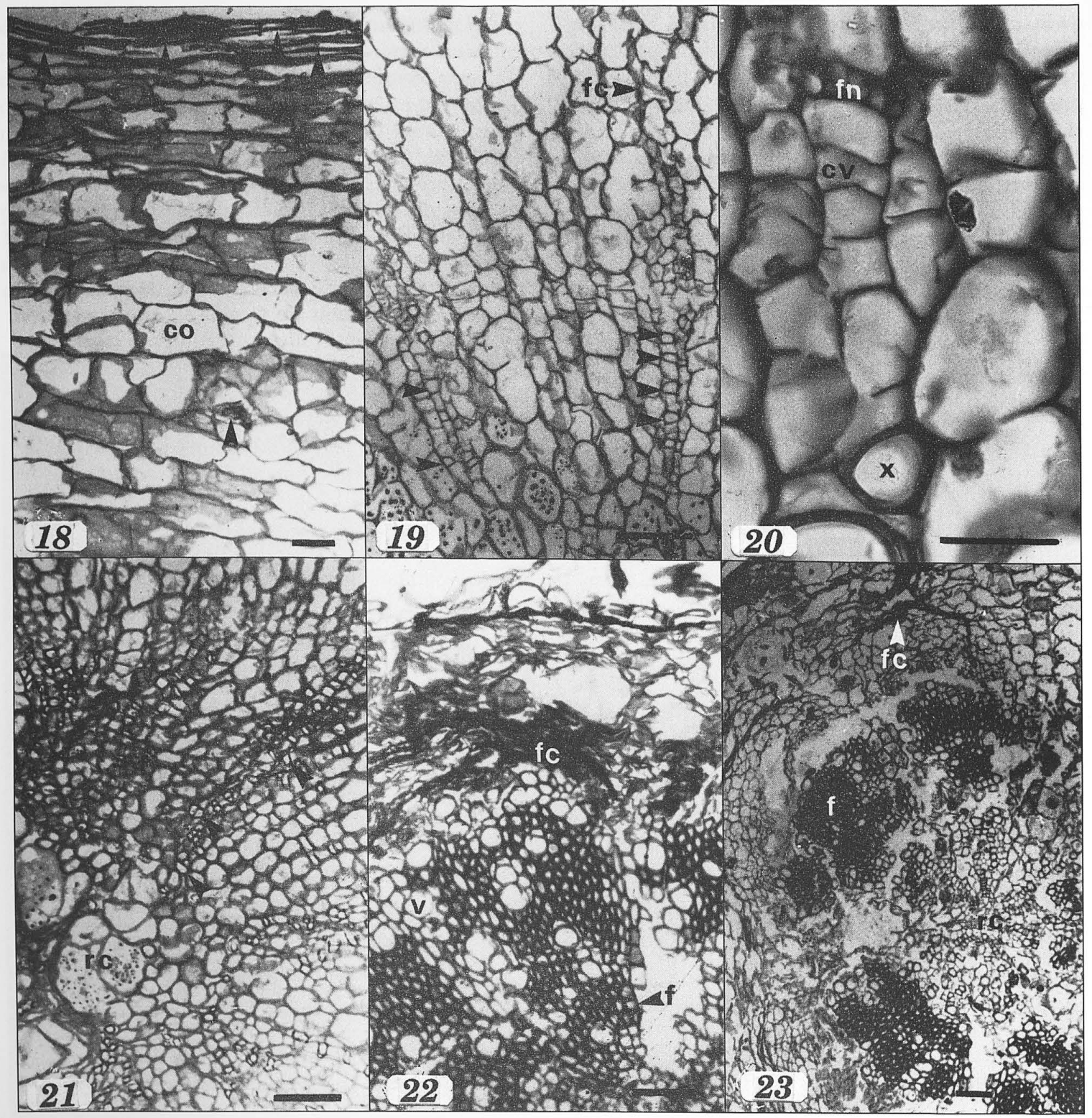

Figuras 18-23. Caracteres anatómicos de la raíz tuberosa y no tuberosa. Cortes transversales. 18-21. Raíz tuberosa. 18. N. lazaro-cardenasii. Peridermis con células de felema no colapsadas (flechas) y haz cortical (flecha). 19. N. lazaro-cardenasii. Xilema y floema secundarios en hileras radiales, vasos y floema colapsado (fechas). 20. N. lazarocardenasii. Zona cambial y elementos de xilema y floema secundarios. 21. N. lazaro-cardenasii. Parenquimatización del metaxilema con abundantes granos de almidón y vasos en hileras radiales (flechas). 22-23. Raíz no tuberosa. 22. N. zopilotensis. Xilema secundario y floema secundario colapsado y no colapsado. 23. N. zopilotensis. Escaso parénquima en la región central de la raíz. $\mathrm{co}=$ córtex; $\mathrm{cv}=$ cámbium vascular; $\mathrm{f}=$ fibras; $\mathrm{fc}=$ floema colapsado; $\mathrm{fn}=$

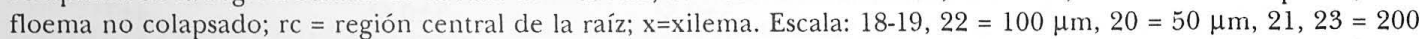
$\mu \mathrm{m}$. 
constituye por células de parénquima de forma isodiamétrica (figura 21), con abundantes granos de almidón y escasas células de mucílago. También se aprecian vasos del tejido vascular primario y secundario dispersos en forma irregular. El incremento generalizado tanto en número como en tamaño de las células de parénquima de esta región, contribuye a aumentar el diámetro de la raíz. En la raíz no tuberosa también se observa un aumento limitado del parénquima del metaxilema en comparación con la raíz tuberosa. Las células de parénquima son de forma irregular (figura 23), no se detectaron células de mucílago, ni otro tipo de contenido; sin embargo sí se observaron escasos vasos y fibras dispersos en esta región.

\section{Discusión}

Los resultados mùestran que las especies de Neoevansia aunque comparten caracteres anatómicos con otros géneros de Cactoideae (cuadro 4) presentan características que las distinguen entre sí. Neoevansia lazarocardenasii y $N$. zopilotensis, por ejemplo, se separan de $N$. striata por el tamaño de las células epidérmicas, el grosor de la hipodermis y algunos caracteres del xilema secundario como son el tipo de punteaduras intervasculares de los elementos de vaso y la longitud de elementos de vaso y fibras. Mientras que $N$. zopilotensis solamente se distingue de $N$. lazaro-cardenasii por el tamaño de las células epidérmicas, el grosor de la hipodermis y el diámetro de los vasos.
El tallo de las especies de Neoevansia tiene cutícula lisa con grosor similar al de algunas especies de Echinocereus, Peniocereus, Wilcoxia y otros géneros de Cactoideae (Gibson y Horak, 1978; Mauseth y Ross, 1988; Loza-Cornejo y Terrazas, 1996, Mauseth et al. 1998). La epidermis es uniestratificada como en la mayoría de las especies de Cactoideae, pero difiere de algunas especies de la tribu Pachycereeae con epidermis múltiples (Terrazas y Loza-Cornejo, 2001). Aunque Marshall (1941) indica que la superficie de las ramas es glabra en Neoevansia, en las tres especies observamos que la superficie es papilosa. Las células epidérmicas tienen papilas unicelulares semejantes a algunas especies de Peniocereus del subgénero Peniocereus. Además, en vista superficial, las células epidérmicas presentan paredes anticlinales rectas, que las distinguen de las especies de Wilcoxia y varias especies de Cactoideae con paredes anticlinales onduladas (Eggli, 1984; Loza-Cornejo y Terrazas, 1996). Es importante resaltar que los estomas se encuentran exclusivamente en las depresiones de las costillas al igual que en especies de Wilcoxia y Peniocereus (LozaCornejo y Terrazas, 1996; Terrazas, com. pers.). La presencia de estomas restringidos a las depresiones de las costillas, posiblemente se relaciona con la aparición tardía de la peridermis. Como sugieren LozaCornejo y Terrazas (1996), dicha distribución podría mantener los estomas funcionales durante varios años. La ausencia de extractivos en las células de la epidermis es un carácter que comparten los géneros Neoevansia, Echinocereus y Wilcoxia; ya que únicamente en algu-

Cuadro 4. Caracteres anatómicos del sistema dérmico y xilema secundario de las especies de Neoevansia, Peniocereus, Wilcoxia y Echinocereus. (Datos tomados de Loza-Cornejo y Terrazas, 1996, Mauseth et al. 1998). $++=$ presente,$-=$ ausente.

\begin{tabular}{lcccc}
\hline Especie & $\begin{array}{c}\text { Papila en } \\
\text { célula } \\
\text { epidérmica }\end{array}$ & $\begin{array}{c}\text { Cristales en } \\
\text { célula } \\
\text { epidérmica }\end{array}$ & $\begin{array}{c}\text { Número de } \\
\text { estratos } \\
\text { hipodermis }\end{array}$ & $\begin{array}{c}\text { Punteaduras } \\
\text { intervasculares } \\
\text { xilema } \\
\text { secundario }\end{array}$ \\
\hline
\end{tabular}

Echinocereus cinerascens

E. pentalophus

E. schmollii

Neoevansia lazaro-cardenasii

N. striata

N. zopilotensis

Peniocereus fosterianus

P. greggii

P. oaxacensis

$P$. viperinus

Wilcoxia albiflora

W. poselgeri

W. tamaulipensis

$\begin{array}{ccc}- & - & 0 \\ - & - & 0 \\ - & - & 0 \\ ++ & - & 1-2 \\ ++ & - & 1 \\ ++ & - & 1-2 \\ - & ++ & 2 \\ ++ & - & 2 \\ - & ++ & >5 \\ ++ & - & 2 \\ - & - & 1 \\ - & - & 1 \\ - & - & 1\end{array}$

$\begin{array}{cc}-- & - \\ -- & - \\ \text { escalariforme } & - \\ \text { alterna } & - \\ \text { escalariforme } & - \\ \text { escalariforme } & ++ \\ \text { alterna } & - \\ \text { escalariforme } & ++ \\ \text { escalariforme } & ++ \\ \text { alterna } & - \\ \text { alterna } & ++/ \\ \text { alterna } & -\end{array}$


nas especies de Peniocereus y otros géneros de Cactoideae es común la presencia de cristales en dichas células (Mauseth, 1996; Porembski, 1996; Mauseth et al. 1998).

La mayoría de los miembros de Cactoideae tienen tallos con una hipodermis colenquimatosa y multiestratificada (Gibson y Horak, 1978; Mauseth, 1996). Sin embargo, el número de estratos y el grosor de la pared varía entre especies de la subfamilia. En las especies de Neoevansia se observó que $N$. lazaro-cardenasii y $N$. zopilotensis se caracterizan por presentar la hipodermis biestratificada, distinguiéndose de $N$. striata que tiene solo un estrato. El grosor de la hipodermis difiere estadísticamente para las tres especies estudiadas, pero se yuxtapone con los valores reportados para especies de Echinocereus, Leptocereus, Peniocereus y Wilcoxia (Loza-Cornejo y Terrazas, 1996; Mauseth et al. 1998). En las especies estudiadas, el córtex ocupa una proporción amplia del tallo, con haces corticales pequeños y abundantes. Mauseth y Sajeva (1992) señalan que los haces corticales probablemente contribuyen en el transporte de agua, azúcares y fotosintatos. Las células de mucílago del córtex son abundantes y de tamaño variable. Es probable que su tamaño esté relacionado con la disponibilidad de agua, ya que se les ha atribuido la función de retención de ésta (Nobel, 1999). Las especies de Neoevansia presentan fibras ubicadas por encima del floema secundario colapsado al igual que los géneros Acanthocereus, Leptocereus y Peniocereus (Gibson y Horak, 1978; Mauseth y Ross, 1988; Mauseth et al. 1998). La médula en especies de Cactoideae se constituye por células parenquimáticas, haces medulares y células de mucílago (Mauseth et al. 1998), sin embargo no se detectaron haces medulares en el material de Neoevansia estudiado, al igual que lo reportado para algunas especies de Acanthocereus, Peniocereus y Wilcoxia (Gibson y Horak, 1978; LozaCornejo y Terrazas, 1996; Mauseth et al. 1998).

$\mathrm{El}$ origen y las características de la peridermis en las especies estudiadas son semejantes a lo reportado para otros miembros de Cactoideae (Mauseth y Ross, 1988; Loza-Cornejo y Terrazas, 1996; Mauseth, 1996). En Cactaceae, particularmente la subfamilia Cactoideae, se ha caracterizado por la presencia de géneros con maderas fibrosas y no fibrosas (Mauseth y Plemons-Rodriguez, 1998). En Neoevansia sólo se observó madera de tipo fibrosa. El xilema secundario forma un cilindro sólido similar a lo reportado para Acanthocereus, Leptocereus, Peniocereus y otros géneros de Cactoideae (Gibson 1973, Mauseth et al. 1998), mientras que para Echinocereus se ha reportado la presencia de madera no fibrosa (Mauseth et al. 1998). El tipo de punteaduras escalariformes y pseudoescalariformes lo presentan $N$. lazaro-cardenasii y $N$. zopilotensis similar a la mayoría de las especies de Peniocereus; pero son diferentes de Neoevansia striata, Peniocereus greggii (Engelm.) Britton et Rose, Wilcoxia albiflora Backeb; W. poselgeri (Lem.) Britton et Rose y W. tamaulipensis Werderm. con punteaduras intervasculares alternas. Las especies de Neoevansia comparten también con algunas especies de Peniocereus la presencia de fibras libriformes no septadas con núcleos prominentes y parénquima axial paratraqueal escaso, pero difieren de Wilcoxia en la presencia de radios. La ausencia de radios representa una especialización del cámbium vascular (Loza-Cornejo y Terrazas, 1996; Terrazas, 2000).

Las raíces de almacenamiento en el material estudiado son del tipo fasciculado tuberoso y también se reportan para especies de los géneros Peniocereus y Wilcoxia (Sánchez-Mejorada, 1973). En raíces tuberosas de Cactoideae, la mayor parte del tejido de almacenamiento se desarrolla en el córtex (Gibson, 1978; Gibson y Nobel, 1986); por lo que contrasta con lo observado en el presente trabajo, ya que en las tres especies estudiadas el aumento de la raíz tuberosa se debe principalmente a la expansión del parénquima radial del xilema y floema secundarios, así como del parénquima del metaxilema en la región central de la raíz, coordinada con las divisiones de las células del córtex. Un proceso de parenquimatización similar se observó en las especies del género Wilcoxia (LozaCornejo y Terrazas, 1996). La raíz tuberosa en comparación con la no tuberosa se distingue por la ausencia de haces corticales, además de la abundancia de fibras y la escasa expansión de los radios en el cilindro vascular.

\section{Implicaciones taxonómicas}

Las comparaciones anatómicas realizadas apoyan las propuestas de Barthlott y Hunt (1993), Hunt (1992) y Taylor (1985) de reagrupar a las tres especies de Neoevansia en el género Peniocereus. Las características morfológicas que comparten $N$. lazaro-cardenasii. $N$. striata y $N$. zopilotensis con los miembros del género Peniocereus son raíces fasciculado tuberosas; tallos provistos de costillas de sección cuneiforme y cantos aplanados, separados por un surco angosto; flores nocturnas, blancas, con receptáculo tubular en la parte inferior e infundibuliforme en la parte superior, con tubo provisto de pocos podarios decurrentes que llevan una escama en cuya axila presentan tricomas, espinas y cerdas. Las características anatómicas que sólo comparten con algunas especies de Peniocereus son: cutícula lisa, apéndices epidérmicos (papilas) unicelulares, forma rectangular y sin contenido en las células epidérmicas, hipodermis colenquimatosa de uno 
o dos estratos, fibras sobre el floema colapsado, porosidad difusa y elementos de vaso con punteaduras intervasculares escalariformes, pseudoescalariformes y alternas. La presencia de tallos monomórficos y papilas epidérmicas en tallo y ramas en las tres especies de Neoevansia permiten sugerir que deben agruparse en el subgénero Peniocereus. Los miembros del género Wilcoxia se distinguen anatómicamente del género Peniocereus por la ausencia de radios en el xilema secundario y de fibras en el floema secundario (Loza-Cornejo y Terrazas, 1996) y del género Echinocereus por la madera no fibrosa y la ausencia de epidermis papilosa (Mauseth et al. 1998).

\section{Conclusiones}

Las especies de Neoevansia comparten características anatómicas con las especies del género Peniocereus y la presencia de tallos monomórficos y papilas epidérmicas con las especies del subgénero Peniocereus. La inclusión de $N$. lazaro-cardenasii, N. striata y N. zopilotensis en el género Peniocereus es más evidente al tomar en cuenta los caracteres anatómicos del sistema dérmico: cutícula lisa, papilas unicelulares, formas rectangulares y sin contenido en las células epidérmicas, hipodermis colenquimatosa con uno y dos estratos; del xilema secundario: porosidad difusa y fibras no septadas, además de que también se caracterizan por presentar madera fibrosa y fibras sobre el floema. Las tres especies de Neoevansia se distinguen entre sí por caracteres anatómicos cuantitativos del tallo como son: largo y ancho de las células epidérmicas, ancho de la hipodermis; diámetro de los vasos, longitud y grosor de la pared de las fibras. Los caracteres anatómicos cualitativos muestran que $N$. striata se distingue por la hipodermis no colenquimatosa de un solo estrato y punteaduras intervasculares alternas en los elementos de vaso del xilema secundario. En cuanto a las raíces tuberosas el incremento en volumen está asociado principalmente al cámbium vascular y parenquimatización del metaxilema en la región central de la raíz y en menor proporción a las divisiones del córtex.

\section{Agradecimientos}

Esta investigación fue apoyada económicamente por CONACYT (Proyecto 0760-N), CONABIO (L074) y Cactus and Succulent Society of America. Agradecemos a Cesario Catalán, Salvador Arias e Hilda Arreola-Nava su ayuda en la colecta de campo y a Miguel Vega el trabajo fotográfico de cuarto obscuro.

\section{Literatura citada}

Bailey I.W. 1954. The use and the abuse of anatomical data in the study of phylogeny and classification. Phytomorphology 1:67-69.

Barthlott W. y Hunt D.R. 1993. Cactaceae. En: Kubitzki K. Ed. The families and genera of vascular plants. SpringerVerlag, Berlín, 161-197.

Boke N.H. 1959. Endomorphic and ectomorphic characters in Pelecyphora and Encephalocarpus. American Journal of Botany 46:197-207.

Bravo-Hollis H. 1978. Las cactáceas de México. 2da. edición. Universidad Nacional Autónoma de México. México. 743 p.

Contreras J.L., Jiménez-R.J., Sánchez-Mejorada H. y Toledo-Manzur C. 1980. Neoevansia lazaro-cardenasii, una nueva especie de cactácea. Cactáceas y Suculentas Mexicanas 25:5154.

Dickison W.C. 1989. Steps toward the natural system of dicotyledons: vegetative anatomy. Aliso 12:555-566.

Eggli U. 1984. Stomatal types of Cactaceae. Plant Systematics and Evolution 146:197-214.

Gibson A.C. 1973. Comparative anatomy of secondary xylem in Cactoideae (Cactaceae). Biotropica 5:29-65.

Gibson A.C. 1978. Structure of Pterocactus tuberosus, a cactus geophyte. Cactus and Succulent Journal 50:41-43.

Gibson A.C. y Horak K.E. 1978. Systematic anatomy and phylogeny of Mexican columnar cacti. Annals of the Missouri Botanical Garden 65:999-1057.

Gibson A.C. y Nobel P.S. 1986. The cactus primer. Harvard University Press, Massachusetts. 286 p.

Hunt D.R. 1992. Cites Cactaceae checklist. Royal Botanic Gardens. Kew, Richmond Surrey. 190 p.

Hunt D.R. y Taylor N.P. 1990. The genera of Cactaceae: progress toward a consensus. Bradleya 8:85-107

Johansen J. 1940. Plant microtechnique. McGraw-Hill Book Co., New York. 523 p.

Loza-Cornejo S. y Terrazas T. 1996. Anatomía del tallo y de la raíz de dos especies de Wilcoxia Britton \& Rose (Cactaceae) del noreste de México. Boletín de la Sociedad Botánica de México 59:13-23.

Marshall W.T. 1941. Cactaceae. 84. (Tomado de SánchezMejorada, 1973).

Mauseth J.D. 1996. Comparative anatomy of tribes Cereeae and Browningieae (Cactaceae). Bradleya 14:66-81.

Mauseth J.D. y Ross R.G. 1988. Systematic anatomy of the primitive cereoid cactus Leptocereus quadricostatus. Bradleya 6:49-64.

Mauseth J.D. y Sajeva M. 1992. Cortical bundles in the persistent photosynthetic stems of cacti. Annals of Botany 70:317-324.

Mauseth J.D. y Plemons-Rodriguez B.J. 1998. Evolution of extreme xeromorphic characters in wood: a study of nine evolutionary lines in Cactaceae. American Journal of Botany 85:209-218. 
Mauseth J.D. Terrazas T. y Loza-Cornejo S. 1998. Anatomy of relictual members of subfamily Cactoideae, IOS Group la (Cactaceae). Bradleya 16:31-43.

Media Cybernetics. 1997. Image-Pro Plus. Version 3.1 for windows. Maryland. 480 p.

Nobel P.S. 1999. Physicochemical and environmental plant physiology. 2nd. ed. Academic Press, San Diego. 474 p.

Nyffeler R. y Eggli U. 1997. Comparative stem anatomy of Eriosyce sensu lato (Cactaceae). Annals of Botany 80:767-786.

Porembski S. 1996. Functional morphology of Aztekium ritteri (Cactaceae); Botanica Acta 109:167-171.

Sánchez-Mejorada H. 1973. El género Neoevansia Marshall, historia y revisión. Cactáceas y Suculentas Mexicanas 18:13-27.

Sánchez-Mejorada H. 1974. Revisión del género Peniocereus. Las cactáceas. Comisión Botánica Exploradora del Estado de México, Toluca. 58 p.
SAS Institute. 1989. SAS procedures guide version 6.04. SAS Institute Inc., Cary. 956 p.

Taylor N.P. 1985. The genus Echinocereus. R. B. G. Kew \& Collingridge, Feltham. 160 p.

Terrazas, T. 2000. Occurrence of perforated ray cells in genera of Pachycereeae (Cactaceae). International Association of Wood Anatomist Journal 21:457-462.

Terrazas, T. y S. Loza-Cornejo. 2001. Phylogenetic relationships of Pachycereeae: a cladistic analysis based on anatomical-morphological data. En: Fleming T.H. y Valiente-Banuet A., Edrs. Evolution, ecology, and conservation of the columnar cacti and their mutualists. Arizona University Press (en prensa). 